\title{
Endometriosis of Rectus Muscle Excised During Cesarean Section: A Case Report and Literature Review
}

\author{
Recep ERİN", Kübra BAKİ ERİN, Derya BURKANKULU AĞIRBAŞ¹, Burcu KEMAL OKATAN² \\ Trabzon, Turkey
}

\begin{abstract}
We aimed to present a case with abdominal wall endometriosis following cesarean section in this case report.

A 32 year old 39 weeks pregnant woman with G2P1 was admitted to gynaecology clinic with abdominal lump and pain in the midline. Her physical examination included a hard and painful palpable subcutaneous mass of $4 \times 5 \mathrm{~cm}$ size in the midline of the abdomen which was semisolid and irreducible.

Under general anesthesia, the mass on the rectus muscle was excised with the healthy tissue around with the diagnosis of endometriosis during cesarean section and the pathological diagnosis was reported as endometriosis.
\end{abstract}

Surgical excision is the best treatment method in abdominal wall endometriosis

Keywords: Endometriosis, Rectus muscle, Cesarean section

Gynecol Obstet Reprod Med 2017;23(1):45-47

\section{Introduction}

Endometriosis was first defined by Von Reclinghausen and Vilar in 1885. It is the presence of the functional endometrial gland and stroma on surfaces other than the uterus, which responds the hormonal stimulation of the ovary and is observed in $15 \%$ of the women with menstruation. Although it is common in the pelvic region, it may be observed in the eye, kidneys, lungs, umbilicus, central nervous system, vesica biliaris, heart, liver, bones, peripheral nerves, skin and abdominal wall. The incidence of endometriosis on the abdominal wall has been reported between 0.03 and $1.08 \%$ in the gynaecological literature. It was first defined by Coley in 1993 (1). Frequently, there is a surgical history, especially caesarean section. It was first reported in 1975 that endometriotic foci may be observed on or around the incision scar after caesarean section or gy-

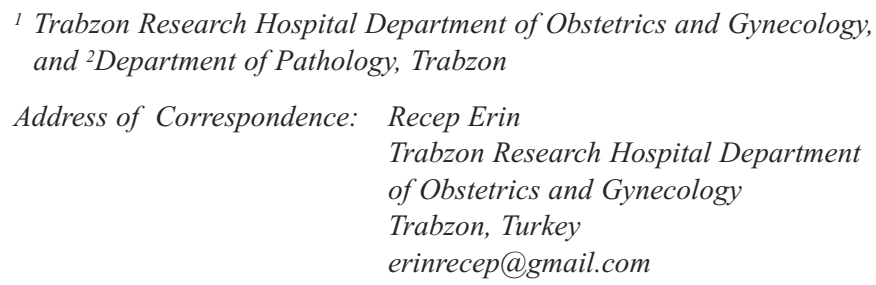

\begin{tabular}{|c|c|}
\hline \multicolumn{2}{|c|}{ Access this article online } \\
\hline $\begin{array}{c}\text { Quick Response Code: } \\
\text { 口: }\end{array}$ & Website: www.gorm.com.tr \\
\cline { 2 - 3 } & DOI: $10.21613 /$ GORM.2016.489 \\
\hline
\end{tabular}

How to cite this article: Erin R. Baki Erin K. Burkankulu Ağırbaş D. Kemal Okutan B. Endometriosis of Rectus Muscle Excised During Cesarean Section: A Case Report and Literature Review. Gynecol Obstet Reprod Med 2017;23(1):45-47 naecological operations (2). The most frequent finding observed in scar endometriosis is palpable mass on surgical incision line; increase in its size may be observed as well as cyclic pain, swelling or cyclic bleeding.

Cases with scar endometriosis or those who are symptomatic are generally admitted to general surgery department. In the study of Jeonghyun et al. on 37 cases with abdominal wall endometriosis, rates of admittance to general surgery and gynaecology clinics were reported to be 30 and $6 \%$ respectively (3). Since its incidence is increased within years and it became a problem met by other specialists as well due to its findings, we aimed to present a case with abdominal wall endometriosis following caesarean section in this case report.

\section{Case Report}

A 32 year old 39 weeks pregnant woman with G2P1 was admitted to gynaecology clinic with abdominal lump and pain in the midline. Her complaints persisted for 1 year, she had a grown mass since then and the pain increased during menstrual cycles. She had a history of caesarean section 3 years before. Her physical examination included a hard and painful palpable subcutaneous mass of $4 \times 5 \mathrm{~cm}$ size in the midline of the abdomen which was semisolid and irreducible. Her gynaecological examination was normal and no specific finding was observed in other system examinations. The anterior abdominal wall MRImagnetic resonance imaging (MRI) examination revealed a $45 \times 50 \mathrm{~mm}$ sized irregular contoured solid mass with heterogeneous appearance which had no relation with the abdominal cavity. Her tumour markers were normal. Benign pathology was considered primarily with regard to her 
history, physical examination and MRI findings, and excision during caesarean section was planned under general anaesthesia. A $4 \times 5 \mathrm{~cm}$ sized fragile, bleeding endometriotic focus was detected on the rectus muscle during caesarean section (Figure 1). Primarily, the pregnancy was terminated properly. Under general anaesthesia, the mass was excised with the healthy tissue around with the diagnosis of endometriosis, and the sample was sent for pathological examination (Figure 2).

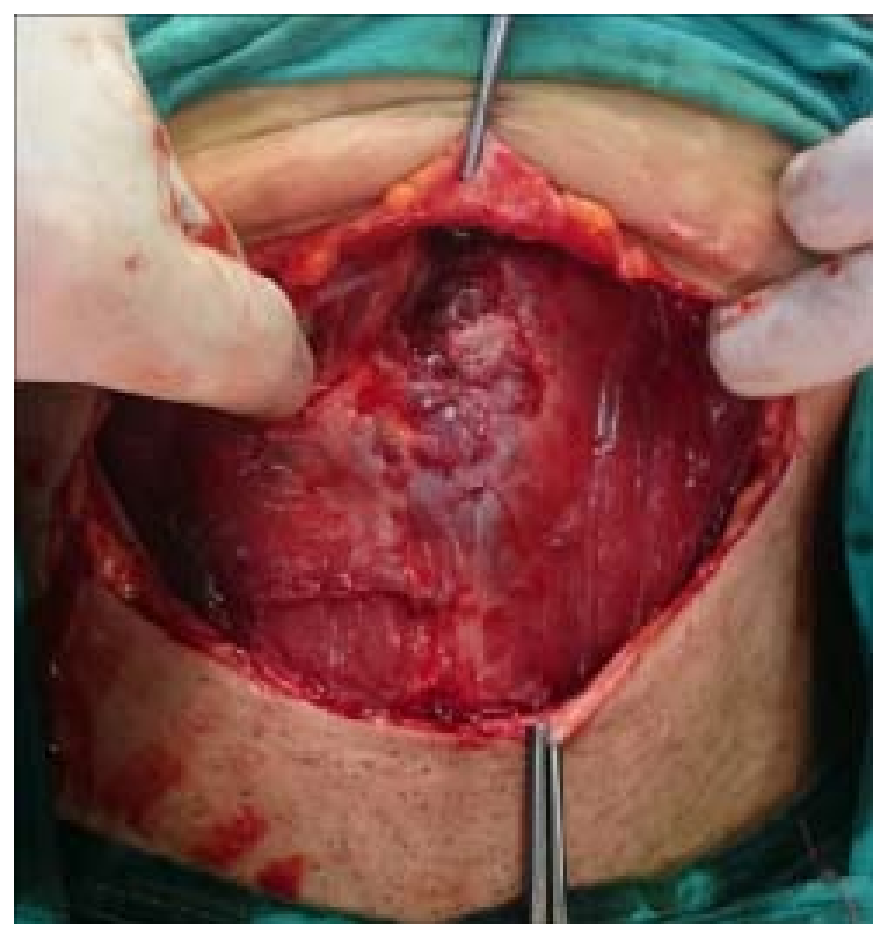

Figure 1: Endometriotic focus on rectus muscle

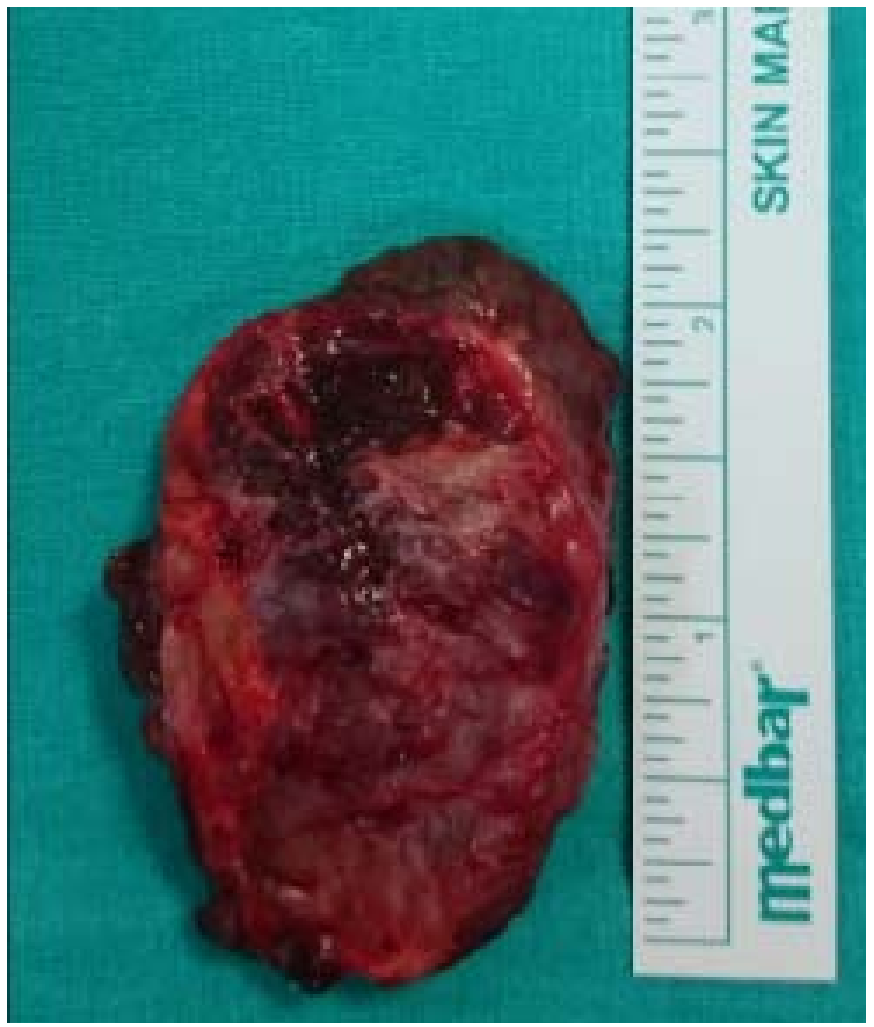

Figure 2: Totally excised endometriotic focus
Histopathological examination revealed endometrial glands and surrounding endometrial stromal tissues within the muscle tissue, and the pathological diagnosis was reported as endometriosis (Figure 3). The case was discharged on the postoperative $2^{\text {nd }}$ day. No early postoperative complication was observed. Mean duration of follow-up was 18 months. No recurrence was observed.

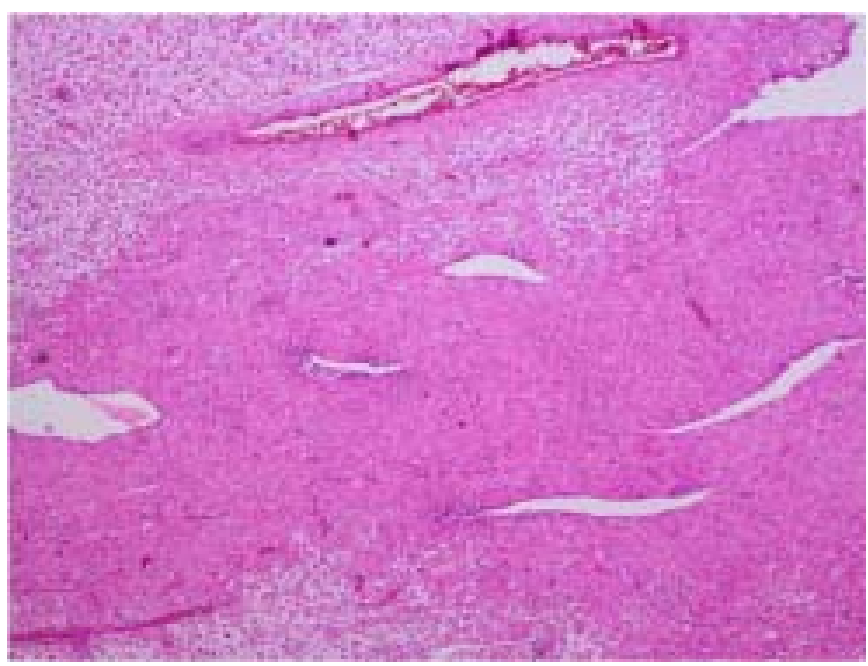

Figure 3: Endometriotic focus formed by decidualized endometrial stroma and cystic glandular endometrial structures between muscle fibres (HEx4)

\section{Discussion}

Extra-pelvic endometriosis consists of $8.9-15 \%$ of all reported endometriosis cases. On the other hand, cases with abdominal wall endometriosis, which is a sub-type of extrapelvic endometriosis, consist of $4 \%$ of all external endometriosis cases (4). The best possible explanation for abdominal wall endometriosis is iatrogenic inoculation during operations. Aimakhu et al. were the first to discover the relation of endometriosis with gynaecological operations (1975) (2). Abdominal wall endometriosis is generally observed within the cutaneous and subcutaneous tissues, but is mainly observed in the incision scar and umbilicus, and is rarely observed in inguinal channel and rectus abdominalis muscle. Abdominal wall endometriosis is observed at a rate of $0.1 \%$ following operations such as cesarean section. In the abdominal wall endometriosis series of Agarwal and Fong including 10 patients, surgical history was detected in 6 patients $(60 \%)$ and spontaneous endometriosis was detected in 4 patients (inguinal channel, umbilicus and rectus abdominis) (5).

Many theories have been put forth in the aetiology of endometriosis. Our case had a history of caesarean section. A history of previous surgery is observed in $20 \%$ of the cases with abdominal wall endometriosis. The endometriotic focus of our patient was within the rectus muscle, distant from the incision. Cases with no abdominal surgery history or those with an endometriotic focus distant from the incision can be explained simply by vascular spread theory. According to that 
theory, endometrial cells access extra genital regions via blood vessels or the lymphatic system, and form endometriotic foci (8). Although our case had a history of caesarean section, it may be accepted as a spontaneous case since the lesion was unrelated to the region of incision and distant from it. Elabsi et al. have reported a case of abdominal wall endometriosis diagnosed 22 years after a caesarean section (6).

The classic finding of abdominal wall endometriosis is lump and pain getting significant during the menstrual period, and cyclic symptoms are present in $50 \%$ of the patients. Hernia, granuloma, hematoma, abscess, lipoma, desmoidal tumour, soft tissue sarcoma, lymphadenopathy and even metastatic tumour should be considered in the differential diagnosis. Accurate preoperative diagnosis of abdominal wall endometriosis varies between $26,7 \%$ and $70 \%$ (3).

Ultrasonography (USG), coloured Doppler USG, computerized tomography (CT) and MRI may provide information about the location, size or density of the mass. USG and MRI are the frequently recommended diagnostic imaging methods. The most common USG finding of the endometriotic mass is hypoechoic, solid mass; but it may also be observed as a cystic or heterogeneous mass. The accuracy of USG in diagnosis has been reported as $80 \%$ (5). CT or MRI may be useful in evaluating the relation of the mass with abdominal cavity. MRI findings can define the hemorrhagic foci within the mass better than other methods depending on the degree and timing of the bleeding. However, precise diagnosis should be made via histopathological examination.

Inflammatory cells and endometrial stroma and glands surrounded by a fibrosis are typically visible within the soft tissue and abdominal wall muscles in histopathological examination. There are studies reporting cases with endometrial tissue based tumours such as clear cell carcinoma, papillary carcinoma, mixed endometrioid and serous carcinoma in the scar endometriosis after caesarean section, as well as those that report rare connective tissue tumours such as desmoid or sarcomas (7). Tissue sampling is performed via thin needle biopsy or total excision as we did. Accuracy of thin needle biopsy is low. Both diagnosis and treatment is performed via total excision. In the abdominal wall endometriosis series of Agarwal et al. including 10 patients, total excision was performed in all cases, and the patients were followed up for one month - three years. According to the post-observational outcomes, total excision has been reported as a curative method (5). The most proper surgical approach has been accepted as the excision of the mass including $1 \mathrm{~cm}$ surrounding tissue, without rupturing the mass.
In 1 of all 4 women with abdominal wall endometriosis, pelvic endometriosis is observed as well. Therefore patients admitted with abdominal wall endometriosis should also be evaluated for possible pelvic endometriosis and treated accordingly. Thus, scar endometriosis recurrence due to the transplantation of the microscopic endometrial tissue residues can be avoided. Reported recurrence rate after total excision is between $0 \%$ and $20 \%(8)$.

As a conclusion, previous gynaecological operation history and increasing lump and pain complaints during menstrual period should be carefully questioned in the evaluation of anterior abdominal wall masses. Achieving the diagnosis and treatment can be earlier by considering endometriosis in differential diagnosis. In order to prevent abdominal wall endometriosis after operations, surgical tools and equipment are recommended to be changed during the closure of the abdomen and irrigation of the abdomen with excessive physiological saline is also important to inhibit the inoculation of the endometrial tissue.

\section{References}

1. Coley BD, Casola G. Incisional endometrioma involving the rectus abdominis muscle and subcutaneous tissues: CT appearance. AJR Am J Roentgenol 1993;160(3):549-50.

2. Aimakhu VE. Anterior abdominal wall endometriosis complicating a uteroabdominal sinus following classical cesarean section. Int Surg 1975;60(12):103-4.

3. Jeonghyun K, Jeong HB, Won SL, Tae HC. Clinical manifestations of abdominal wall endometriosis: a single center experience. Arch Gynecol Obstet 2002;286 (3):2565-2.

4. Douglas C, Rotimi O. Extragenital endometriosis - a clinicopathological review of a Glasgow hospital experience with case illustrations. J Obstet Gynaecol 2004;24(11): 804-8.

5. Agarwal A, Fong YF. Cutaneous endometriosis. Singapore Med J 2008;9(8):704-7.

6. Elabsi M, Lahlou MK, Rouas L, Essadel H, Benamer S, Mohammadi A, et al. Cicatrix endometriosis of the abdominal wall. Ann Chir 2002;127(1):65-7.

7. Miller DM, Schouls JJ, Ehlen TG. Clear cell carcinoma arisingin extragonadal endometriosis in a caesarean section scar during pregnancy. Gynecol Oncol 1998;70(1): 127-30.

8. Merran S, Karila-Cohen P. Incisional subcutaneous endometrioma of the abdominal wall: report of two cases. J Radiol 2004;85(4 Pt 1):409-10. 